\title{
Preservation of the Exchange Principle via Lattice Operations on $(\mathrm{S}, \mathrm{N})-$ Implications
}

\author{
Dimitrios S. Grammatikopoulos(i) and Basil K. Papadopoulos ${ }^{(凶)}(\mathbb{D}$ \\ Section of Mathematics and Informatics, Department of Civil Engineering, \\ School of Engineering, Democritus University of Thrace, 67100 Kimeria, Greece \\ \{dimigram2, papadob\}@civil.duth.gr
}

\begin{abstract}
In this paper, we investigate a special case of an open problem that is related to the exchange principle, a property of fuzzy implications. We focus on the cases of $(\mathrm{S}, \mathrm{N})$ - implications and the preservation of the exchange principle via lattice operations. We present and prove some sufficient conditions such that the exchange principle is preserved under the join and meet operations if we use $(\mathrm{S}, \mathrm{N})$ - implications.
\end{abstract}

Keywords: (S,N)- implication · Exchange principle

\section{Introduction}

The applications of fuzzy logic were the uprising of the automata theory, robotics, approximate reasoning, image processing, pattern recognition, artificial intelligence and many other scientific and applicable areas. The transition of the two valued $\{0,1\}$ - logic to the close $[0,1]$ - logic was the total revolution from the second half of the twentieth century to the present and as it appears in the future. Many definitions in fuzzy logic are generalizations from the classical ones. Fuzzy implications, their properties and some of their construction methods are also such generalizations (see [1]).

Some of them are $(\mathrm{S}, \mathrm{N})$ - implications, which are based on the following classical tautology

$$
(p \Rightarrow q) \equiv\left(p^{\prime} \vee q\right)
$$

and the property exchange principle of fuzzy implications, which is based on the following classical tautology

$$
[p \Rightarrow(q \Rightarrow r)] \equiv[q \Rightarrow(p \Rightarrow r)]
$$

In this paper we investigate a special case of an open problem, that was addressed by Baczyński and Jayaram in 2008 (see [1] Remark 6.1.5). It was also formulated by Mesiar and Stupňanová in 2015 (see [6] Problem 3.1). This is the following: 
Characterize the subfamily of all fuzzy implications $((\mathrm{S}, \mathrm{N})-$ implications,

R-implications, etc.) which preserve the (EP) for lattice operations.

Although this problem has been investigated by Vemuri and Jayaram in [8,9], it is not fully solved. They investigated it, in general for any two fuzzy implications. In their results are contained some sufficient conditions for two fuzzy implications such that, $(\mathrm{EP})$ is preserved via the lattice operations (see Section 3 in $[8,9]$ ). They also found and necessary ones, but under some conditions (see Section 4 in [8,9]). Furthermore, in [5] a generation of fuzzy implications with specific properties was succeeded, but not for the property (EP). All of these were the motivation for this paper. All efforts have been done for any fuzzy implications, but not extensively for a subfamily of them. Thus, we investigate it again only for $(\mathrm{S}, \mathrm{N})$-implications. The main reason of the choice of this subfamily is that $(\mathrm{S}, \mathrm{N})$-implications satisfy $(\mathrm{EP})$. The following work will appear new conditions for the preservation of the exchange principle in the case we use $(\mathrm{S}, \mathrm{N})$-implications.

\section{Preliminaries}

Definition 1. [1,3,4]. A decreasing function $N:[0,1] \rightarrow[0,1]$ is called fuzzy negation, if $N(0)=1$ and $N(1)=0$.

Remark 1. (i) (Example 1.4.4 in [1]) The so called, classical fuzzy negation is

$$
N_{C}(x)=1-x
$$

(ii) Moreover, the crisp fuzzy negations (see Remark 2.1 in [2]) are

$$
\begin{gathered}
N^{\alpha}(x)=\left\{\begin{array}{l}
0, \text { if } x \geq \alpha \\
1, \text { if } x<\alpha
\end{array}, \text { where } \alpha \in(0,1]\right. \text { and } \\
N_{\alpha}(x)=\left\{\begin{array}{l}
0, \text { if } x>\alpha \\
1, \text { if } x \leq \alpha
\end{array}, \text { where } \alpha \in[0,1) .\right.
\end{gathered}
$$

Definition 2. (Definition 2.1.1 in [1]). A function $T:[0,1]^{2} \rightarrow[0,1]$ is called a triangular norm (shortly t-norm) if it satisfies, for all $x, y, z \in[0,1]$, the following conditions (Table 1)

$$
\begin{gathered}
T(x, y)=T(y, x), \\
T(x, T(y, z))=T(T(x, y), z),
\end{gathered}
$$

if $y \leq z$, then $T(x, y) \leq T(x, z)$, i.e., $T(x, \cdot)$ is increasing,

$$
T(x, 1)=x
$$


Definition 3. (Definition 2.2.1 in [1]). A function $S:[0,1]^{2} \rightarrow[0,1]$ is called a triangular conorm (shortly $t$-conorm), if it satisfies, for all $x, y, z \in[0,1]$, the following conditions

$$
\begin{gathered}
S(x, y)=S(y, x), \\
S(x, S(y, z))=S(S(x, y), z), \\
\text { if } y \leq z \text {, then } S(x, y) \leq S(x, z) \text {, i.e., } S(x, \cdot) \text { is increasing, } \\
S(x, 0)=x .
\end{gathered}
$$

Table 1. Examples of t-conorms (Table 2.2 in [1]).

\begin{tabular}{l|l}
\hline Name & Formula \\
\hline Maximum & $S_{M}=\max \{x, y\}$ \\
\hline Probor & $S_{P}=x+y-x \cdot y$ \\
\hline Nilpotent maximum & $S_{n M}= \begin{cases}1, & \text { if } x+y \geq 1 \\
\max \{x, y\}, & \text { otherwise }\end{cases}$ \\
\hline
\end{tabular}

Remark 2. (Table 2.2 in [1]). In this work we use the minimum t-norm, which has the following formula

$$
T_{M}=\min \{x, y\}, x, y \in[0,1]
$$

Definition 4. (Definition 1.1 .1 in [1]). A function $I:[0,1]^{2} \rightarrow[0,1]$ is called a fuzzy implication if

$I$ is decreasing with respect to the first variable,

I is increasing with respect to the second variable,

$$
\begin{aligned}
& I(0,0)=1, \\
& I(1,1)=1, \\
& I(1,0)=0 .
\end{aligned}
$$


Definition 5. (Definition 1.3.1(ii) in [1]). A fuzzy implication I is said to satisfy the exchange principle, if

$$
I(x, I(y, z))=I(y, I(x, z)), x, y, z \in[0,1] .
$$

Definition 6. (Definition 2.4.1 in [1]). A function $I:[0,1]^{2} \rightarrow[0,1]$ is called an $(S, N)$ - implication if there exist a t-conorm $S$ and a fuzzy negation $N$ such that

$$
I(x, y)=S(N(x), y), x, y \in[0,1]
$$

Moreover, if $I$ is an $(S, N)$ - implication generated from $S$ and $N$, then we will often denote it by $I_{S, N}$.

Proposition 1. (Proposition 2.4.3(i) in [1]). If $I_{S, N}$ is an $(S, N)$ - implication, then it satisfies (EP) (Table 2).

Table 2. Examples of (S,N)- implications (Tables 1.3 and 2.4 in [1]).

\begin{tabular}{l|l|l|l}
\hline Implication's name & $\mathrm{S}$ & $\mathrm{N}$ & $(\mathrm{S}, \mathrm{N})-$ implication \\
\hline Kleene-Dienes & $S_{M}$ & $N_{C}$ & $I_{K D}(x, y)=\max \{1-x, y\}$ \\
\hline Reichenbach & $S_{P}$ & $N_{C}$ & $I_{R C}(x, y)=1-x+x \cdot y$ \\
\hline Fodor & $S_{n M}$ & $N_{C}$ & $I_{F D}(x, y)= \begin{cases}1, & \text { if } x \leq y \\
\max \{1-x, y\}, & \text { if } x>y\end{cases}$ \\
\hline
\end{tabular}

Moreover, for any t-conorm $S$ there are the following $(\mathrm{S}, \mathrm{N})$ - implications

$$
\begin{gathered}
I_{S, N^{\alpha}}(x, y)=\left\{\begin{array}{l}
y, \text { if } x \geq \alpha \\
1, \text { if } x<\alpha
\end{array}, \text { where } \alpha \in(0,1]\right. \text { and } \\
I_{S, N_{\alpha}}(x)=\left\{\begin{array}{l}
y, \text { if } x>\alpha \\
1, \text { if } x \leq \alpha
\end{array}, \text { where } \alpha \in[0,1) .\right.
\end{gathered}
$$

The lattice theory is well known by the literature. In this work we only need the lattice operations (join and meet) that are defined by Baczyński and Jayaram in Theorem 6.1.1 in [1]. Although, we will not deal with the lattice theory, we must present the preliminaries to make the problem just understandable. Let us consider as $\mathcal{F} \mathcal{I}$ be the family of all fuzzy implications and the partial order $\leq$ induced from the unit interval $[0,1]$.

Theorem 1. (Theorem 6.1.1 in [1]). The family $(\mathcal{F I}, \leq)$ is a complete, completely distributive lattice with the lattice operations

$$
\begin{aligned}
& (I \bigvee J)(x, y)=\max \{I(x, y), J(x, y)\}=S_{M}(I(x, y), J(x, y)), x, y \in[0,1] \\
& (I \bigwedge J)(x, y)=\min \{I(x, y), J(x, y)\}=T_{M}(I(x, y), J(x, y)), x, y \in[0,1]
\end{aligned}
$$

where $I, J \in \mathcal{F} \mathcal{I}$. 


\section{Main Results}

As we have mentioned before, we focus in the Problem 3.1 in [6], in the specific case we use $(\mathrm{S}, \mathrm{N})$ - implications. According to Proposition 1, it is known that $(\mathrm{S}, \mathrm{N})$ - implications always satisfy $(\mathrm{EP})$. So, the problem we investigate is the following:

Problem 3.1 (a special case): Let $I_{S, N}$ and $J_{S, N}$ be two (S,N)-implications, not necessarily generated from the same t-conorm $S$ and fuzzy negation $N$. Are $I_{S, N} \bigvee J_{S, N}$ and $I_{S, N} \bigwedge J_{S, N}$ satisfy (EP)? If not, what are the conditions of the preservation of $(\mathrm{EP})$ ?

Firstly we present the following lemmas.

Lemma 1. (Example 2.1.(i) in [8] and Example 2.2.(i) in [9]). Let I, J be two comparable fuzzy implications that satisfy $(E P)$, then $I \bigvee J$ and $I \bigwedge J$ satisfy $(E P)$.

Proof. Without loss of generality, we assume that $I \leq J$. The proof is obvious, since $I \bigvee J=J$ and $I \bigwedge J=I$.

Lemma 2. Let $I_{S_{1}, N_{1}}$ and $I_{S_{2}, N_{2}}$ be two $(S, N)$ - implications generated from comparable t-conorms and fuzzy negations, such that $S_{1} \leq S_{2}$ and $N_{1} \leq N_{2}$. Then, the fuzzy implications $I_{S_{1}, N_{1}} \bigvee I_{S_{2}, N_{2}}$ and $I_{S_{1}, N_{1}} \wedge I_{S_{2}, N_{2}}$ satisfy (EP).

Proof. Just notify that $I_{S_{1}, N_{1}} \leq I_{S_{2}, N_{2}}$ and the proof is deduced by Lemma 1 .

A special case of Lemma 2 is the following.

Lemma 3. Let $I_{S_{1}, N}$ and $I_{S_{2}, N}$ be two $(S, N)$ - implications generated from the same fuzzy negation $N$ and two comparable t-conorms. Then, the fuzzy implications $I_{S_{1}, N} \bigvee I_{S_{2}, N}$ and $I_{S_{1}, N} \bigwedge I_{S_{2}, N}$ satisfy $(E P)$.

Proof. It is deduced by Lemma 2.

Lemma 4. For all $x, y, z, w \in[0,1]$ it is

$$
\begin{gathered}
S_{M}\left(S_{M}(x, y), S_{M}(z, w)\right)=\max \{x, y, z, w\} \text { and } \\
T_{M}\left(T_{M}(x, y), T_{M}(z, w)\right)=\min \{x, y, z, w\} .
\end{gathered}
$$

Proof. The proof is omitted due to its simplicity.

Let us study the case that, $I_{S, N}$ and $J_{S, N}$ generated from the same t-conorm $S$. 
Theorem 2. Let $I_{S, N_{1}}$ and $I_{S, N_{2}}$ be two $(S, N)$-implications generated from the same t-conorm $S$. Then, the fuzzy implications $I_{S, N_{1}} \bigvee I_{S, N_{2}}$ and $I_{S, N_{1}} \wedge I_{S, N_{2}}$ satisfy (EP).

Proof. For all $x, y, z \in[0,1]$ it is

$$
\begin{aligned}
& I_{S, N_{1}}\left(x,\left(I_{S, N_{1}} \bigvee I_{S, N_{2}}\right)(y, z)\right) \\
& =I_{S, N_{1}}\left(x, S_{M}\left(I_{S, N_{1}}(y, z), I_{S, N_{2}}(y, z)\right)\right) \\
& =I_{S, N_{1}}\left(x, \max \left\{I_{S, N_{1}}(y, z), I_{S, N_{2}}(y, z)\right\}\right) \\
& \stackrel{(I 2)}{=} \max \left\{I_{S, N_{1}}\left(x, I_{S, N_{1}}(y, z)\right), I_{S, N_{1}}\left(x, I_{S, N_{2}}(y, z)\right)\right\} \\
& =S_{M}\left(I_{S, N_{1}}\left(x, I_{S, N_{1}}(y, z)\right), I_{S, N_{1}}\left(x, I_{S, N_{2}}(y, z)\right)\right) .
\end{aligned}
$$

Moreover,

$$
\begin{aligned}
I_{S, N_{1}}\left(x, I_{S, N_{2}}(y, z)\right) & =S\left(N_{1}(x), S\left(N_{2}(y), z\right)\right) \\
& \stackrel{(S 1)}{=} S\left(N_{1}(x), S\left(z, N_{2}(y)\right)\right) \\
& \stackrel{(S 2)}{=} S\left(S\left(N_{1}(x), z\right), N_{2}(y)\right) \\
& \stackrel{(S 1)}{=} S\left(N_{2}(y), S\left(N_{1}(x), z\right)\right) \\
& =I_{S, N_{2}}\left(y, I_{S, N_{1}}(x, z)\right) .
\end{aligned}
$$

Furthermore, $I_{S, N_{1}}$ satisfies $(\mathrm{EP})$. So, we conclude that,

$$
\begin{aligned}
& I_{S, N_{1}}\left(x,\left(I_{S, N_{1}} \bigvee I_{S, N_{2}}\right)(y, z)\right) \\
& =S_{M}\left(I_{S, N_{1}}\left(x, I_{S, N_{1}}(y, z)\right), I_{S, N_{1}}\left(x, I_{S, N_{2}}(y, z)\right)\right) \\
& =S_{M}\left(I_{S, N_{1}}\left(y, I_{S, N_{1}}(x, z)\right), I_{S, N_{2}}\left(y, I_{S, N_{1}}(x, z)\right)\right)
\end{aligned}
$$

By swapping $N_{1}$ and $N_{2}$ it turns out the following equation

$$
\begin{aligned}
& I_{S, N_{2}}\left(x,\left(I_{S, N_{2}} \bigvee I_{S, N_{1}}\right)(y, z)\right. \\
& \quad=S_{M}\left(I_{S, N_{2}}\left(y, I_{S, N_{2}}(x, z)\right), I_{S, N_{1}}\left(y, I_{S, N_{2}}(x, z)\right)\right) .
\end{aligned}
$$

It is obvious by (S1) that,

$$
I_{S, N_{2}}\left(x,\left(I_{S, N_{1}} \bigvee I_{S, N_{2}}\right)(y, z)\right)=I_{S, N_{2}}\left(x,\left(I_{S, N_{2}} \bigvee I_{S, N_{1}}\right)(y, z)\right)
$$


So,

$$
\begin{aligned}
& \left(I_{S, N_{1}} \bigvee I_{S, N_{2}}\right)\left(x,\left(I_{S, N_{1}} \bigvee I_{S, N_{2}}\right)(y, z)\right) \\
& =S_{M}\left(I_{S, N_{1}}\left(x,\left(I_{S, N_{1}} \bigvee I_{S, N_{2}}\right)(y, z)\right), I_{S, N_{2}}\left(x,\left(I_{S, N_{1}} \bigvee I_{S, N_{2}}\right)(y, z)\right)\right) \\
& =S_{M}\left(S_{M}\left(I_{S, N_{1}}\left(y, I_{S, N_{1}}(x, z)\right), I_{S, N_{2}}\left(y, I_{S, N_{1}}(x, z)\right)\right),\right. \\
& \left.\quad S_{M}\left(I_{S, N_{2}}\left(y, I_{S, N_{2}}(x, z)\right), I_{S, N_{1}}\left(y, I_{S, N_{2}}(x, z)\right)\right)\right) \\
& \stackrel{(12))}{=} \max \left\{I_{S, N_{1}}\left(y, I_{S, N_{1}}(x, z)\right), I_{S, N_{2}}\left(y, I_{S, N_{1}}(x, z)\right), I_{S, N_{2}}\left(y, I_{S, N_{2}}(x, z)\right), \quad I_{S, N_{1}}\left(y, I_{S, N_{2}}(x, z)\right)\right\} \\
& =\max \left\{I_{S, N_{1}}\left(y, I_{S, N_{1}}(x, z)\right), I_{S, N_{1}}\left(y, I_{S, N_{2}}(x, z)\right), I_{S, N_{2}}\left(y, I_{S, N_{2}}(x, z)\right),\right. \\
& \stackrel{(12)}{=} S_{M}\left(S_{M}\left(I_{S, N_{1}}\left(y, I_{S, N_{1}}(x, z)\right), I_{S, N_{1}}\left(y, I_{S, N_{2}}(x, z)\right)\right)\right) \\
& \left.\quad S_{M}\left(I_{S, N_{2}}\left(y, I_{S, N_{2}}(x, z)\right), I_{S, N_{2}}\left(y, I_{S, N_{1}}(x, z)\right)\right)\right) \\
& \stackrel{\left(I_{2}\right)}{=} S_{M}\left(I_{S, N_{1}}\left(y, S_{M}\left(I_{S, N_{1}}(x, z) I_{S, N_{2}}(x, z)\right)\right),\right. \\
& \left.\quad I_{S, N_{2}}\left(y, S_{M}\left(I_{S, N_{2}}(x, z), I_{S, N_{1}}(x, z)\right)\right)\right) \\
& =S_{M}\left(I_{S, N_{1}}\left(y,\left(I_{S, N_{1}} \bigvee I_{S, N_{2}}\right)(x, z)\right), I_{S, N_{2}}\left(y,\left(I_{S, N_{1}} \bigvee I_{S, N_{2}}\right)(x, z)\right)\right) \\
& =\left(I_{S, N_{1}} \bigvee I_{S, N_{2}}\right)\left(y,\left(I_{S, N_{1}} \bigvee I_{S, N_{2}}\right)(x, z)\right)
\end{aligned}
$$

thus, $I_{S, N_{1}} \bigvee I_{S, N_{2}}$ satisfies (EP).

The proof for the meet is similar, therefore it is omitted.

The same result does not hold in general, when $I_{S, N}$ and $J_{S, N}$ generated from the same fuzzy negation $N$. In the case we use two comparable t-conorms the lattice operations preserve (EP), according to Lemma 3. On the other hand, the preservation of (EP) is not ensured if we use two not comparable t-conorms. The proof is the following counterexample.

Example 1. Consider the fuzzy implications $I_{S_{n M}, N_{C}}=I_{F D}$ and $I_{S_{P}, N_{C}}=I_{R C}$. The t-conorms $S_{n M}$ and $S_{P}$ are not comparable since

$$
\begin{aligned}
& S_{n M}(0.2,0.3)=0.3<0.44=S_{P}(0.2,0.3) \text { and } \\
& S_{n M}(0.2,0.9)=1>0.92=S_{P}(0.2,0.9) .
\end{aligned}
$$

Moreover, it is

$$
\begin{aligned}
\left(I_{F D} \bigvee I_{R C}\right)(x, y) & =S_{M}\left(I_{F D}(x, y), I_{R C}(x, y)\right) \\
& = \begin{cases}1, & \text { if } x \leq y \\
\max \{1-x, y, 1-x+x \cdot y\}, & \text { if } x>y\end{cases} \\
& = \begin{cases}1, & \text { if } x \leq y \\
1-x+x \cdot y, & \text { if } x>y\end{cases}
\end{aligned}
$$


and

$$
\begin{aligned}
\left(I_{F D} \bigwedge I_{R C}\right)(x, y) & =T_{M}\left(I_{F D}(x, y), I_{R C}(x, y)\right) \\
& =\min \left\{I_{F D}(x, y), I_{R C}(x, y)\right\} \\
& = \begin{cases}1-x+x \cdot y, & \text { if } x \leq y \\
\min \{\max \{1-x, y\}, 1-x+x \cdot y\}, & \text { if } x>y\end{cases} \\
& = \begin{cases}1-x+x \cdot y, & \text { if } x \leq y \\
1-x, & \text { if } y<x<1-y \\
y, & \text { if } 1-x<y<x\end{cases}
\end{aligned}
$$

Both, $I=I_{F D} \bigvee I_{R C}$ and $J=I_{F D} \bigwedge I_{R C}$ violate $(\mathrm{EP})$, since

$$
\begin{aligned}
& I(0.8, I(0.3,0.2))=0.808 \neq 1=I(0.3, I(0.8,0.2)) \text { and } \\
& J(0.2, J(0.8,0.3))=0.86 \neq 0.888=J(0.8, J(0.2,0.3)) .
\end{aligned}
$$

Moreover, when $I_{S, N}$ and $J_{S, N}$ generated from two comparable t-conorms and two comparable fuzzy negations the lattice operations preserve (EP), according to Lemma 3. On the other hand, the preservation of (EP) is not ensured if we use two not comparable t-conorms or fuzzy negations respectively. This will be proved in the following counterexamples.

Example 2. Consider the fuzzy implications $I_{S_{n M}, N_{C}}=I_{F D}$ and

$$
\begin{aligned}
I_{S_{P}, N_{K}} & =S_{P}\left(N_{K}(x), y\right) \\
& =N_{K}(x)+y-N_{K}(x) \cdot y \\
& =\left(1-x^{2}\right)+y-\left(1-x^{2}\right) \cdot y \\
& =1-x^{2}+x^{2} \cdot y,
\end{aligned}
$$

where $N_{K}(x)=1-x^{2}$ (see [1] Table 1.6). It is obvious that $N_{C} \leq N_{K}$. On the other hand, the t-conorms $S_{n M}$ and $S_{P}$ are not comparable (see Example 1). Moreover, it is

$$
\begin{aligned}
\left(I_{F D} \bigvee I_{S_{P}, N_{K}}\right)(x, y) & =S_{M}\left(I_{F D}(x, y), I_{S_{P}, N_{K}}(x, y)\right) \\
& = \begin{cases}1, & \text { if } x \leq y \\
\max \left\{1-x, y, 1-x^{2}+x^{2} \cdot y\right\}, & \text { if } x>y\end{cases} \\
& = \begin{cases}1, & \text { if } x \leq y \\
1-x^{2}+x^{2} \cdot y, & \text { if } x>y\end{cases}
\end{aligned}
$$

and

$$
\begin{aligned}
\left(I_{F D} \bigwedge I_{S_{P}, N_{K}}\right)(x, y) & =T_{M}\left(I_{F D}(x, y), I_{S_{P}, N_{K}}(x, y)\right) \\
& =\min \left\{I_{F D}(x, y), I_{S_{P}, N_{K}}(x, y)\right\} \\
& = \begin{cases}1-x^{2}+x^{2} \cdot y, & \text { if } x \leq y \\
\min \left\{\max \{1-x, y\}, 1-x^{2}+x^{2} \cdot y\right\}, & \text { if } x>y\end{cases}
\end{aligned}
$$




$$
\begin{aligned}
& = \begin{cases}1-x^{2}+x^{2} \cdot y, & \text { if } x \leq y \\
1-x, & \text { if } y<x<1-y \\
\min \left\{y, 1-x^{2}+x^{2} \cdot y\right\}, & \text { if } 1-x<y<x\end{cases} \\
& = \begin{cases}1-x^{2}+x^{2} \cdot y, & \text { if } x \leq y \\
1-x, & \text { if } y<x<1-y \\
y, & \text { if } 1-x<y<x\end{cases}
\end{aligned}
$$

Both, $I=I_{F D} \bigvee I_{S_{P}, N_{K}}$ and $J=I_{F D} \wedge I_{S_{P}, N_{K}}$ violate $(\mathrm{EP})$, since

$$
\begin{aligned}
& I(0.8, I(0.5,0.2))=1 \neq 0.872=I(0.5, I(0.8,0.2)) \text { and } \\
& J(0.2, J(0.8,0.3))=0.972 \neq 0.98208=J(0.8, J(0.2,0.3)) .
\end{aligned}
$$

Example 3. Consider the fuzzy implication $I_{S_{M}, N_{C}}=I_{K D}$. Let the fuzzy negation

$$
N_{E x 3}(x)= \begin{cases}1, & \text { if } x \in[0,0.5) \\ 0.3, & \text { if } x \in[0.5,1) \\ 0, & \text { if } x=1\end{cases}
$$

and the corresponding $(\mathrm{S}, \mathrm{N})-$ implication

$$
\begin{aligned}
I_{S_{P}, N_{E x 3}} & =S_{P}\left(N_{E x 3}(x), y\right) \\
& =N_{E x 3}(x)+y-N_{E x 3}(x) \cdot y \\
& = \begin{cases}1, & \text { if } x \in[0,0.5) \\
0.3+0.7 \cdot y & \text { if } x \in[0.5,1) \\
y, & \text { if } x=1\end{cases}
\end{aligned}
$$

It is known that $S_{M} \leq S_{P}$ (see [1] Remark 2.2.5 (viii)). On the other hand the fuzzy negations $N_{C}$ and $N_{E x 3}$ are not comparable. Moreover, it is

$$
\begin{aligned}
\left(I_{K D} \bigvee I_{S_{P}, N_{E x 3}}\right)(x, y) & =S_{M}\left(I_{K D}(x, y), I_{S_{P}, N_{E x 3}}(x, y)\right) \\
& = \begin{cases}\max \{1-x, y, 0.3+0.7 \cdot y\} & \text { if } x \in[0.5,1) \\
y, & \text { if } x=1 \\
1, & \text { otherwise }\end{cases} \\
& = \begin{cases}\max \{1-x, 0.3+0.7 \cdot y\} & \text { if } x \in[0.5,1) \\
y, & \text { if } x=1 \\
1, & \text { otherwise }\end{cases}
\end{aligned}
$$

and

$$
\begin{aligned}
& \left(I_{K D} \bigwedge I_{S_{P}, N_{E x 3}}\right)(x, y)=T_{M}\left(I_{K D}(x, y), I_{S_{P}, N_{E x 3}}(x, y)\right) \\
& = \begin{cases}\min \{\max \{1-x, y\}, 1\}, & \text { if } x \in[0,0.5) \\
\min \{\max \{1-x, y\}, 0.3+0.7 \cdot y\}, & \text { if } x \in[0.5,1) \\
\min \{\max \{1-x, y\}, y\}, & \text { if } x=1\end{cases} \\
& = \begin{cases}\max \{1-x, y\}, & \text { if } x \in[0,0.5) \\
\min \{\max \{1-x, y\}, 0.3+0.7 \cdot y\} & \text { if } x \in[0.5,1) \\
y, & \text { if } x=1\end{cases}
\end{aligned}
$$


Both, $I=I_{K D} \bigvee I_{S_{P}, N_{E x 3}}$ and $J=I_{K D} \wedge I_{S_{P}, N_{E x 3}}$ violate (EP), since

$$
\begin{aligned}
& I(0.8, I(0.5,0.2))=0.65 \neq 0.608=I(0.5, I(0.8,0.2)) \text { and } \\
& J(0.8, J(0.5,0.1))=0.37 \neq 0.44=J(0.5, J(0.8,0.1)) .
\end{aligned}
$$

Example 4. Consider the fuzzy implications $I_{S_{n M}, N_{C}}=I_{F D}$ and

$$
I_{S_{P}, N_{E x 3}}= \begin{cases}1, & \text { if } x \in[0,0.5) \\ 0.3+0.7 \cdot y, & \text { if } x \in[0.5,1) \\ y, & \text { if } x=1\end{cases}
$$

The t-conorms $S_{n M}$ and $S_{P}$ are not comparable (see Example 1). The same holds for the fuzzy negations $N_{C}$ and $N_{E x 3}$. Moreover, it is

$$
\begin{aligned}
\left(I_{F D} \bigvee I_{S_{P}, N_{E x 3}}\right)(x, y)=S_{M}\left(I_{F D}(x, y), I_{S_{P}, N_{E x 3}}(x, y)\right) \\
= \begin{cases}\max \{1-x, y, 0.3+0.7 \cdot y\} & \text { if } x \in[0.5,1) \text { and } x>y \\
y, & \text { if } x=1 \\
1, & \text { otherwise }\end{cases} \\
= \begin{cases}\max \{1-x, 0.3+0.7 \cdot y\}, & \text { if } x \in[0.5,1) \text { and } x>y \\
y, & \text { if } x=1 \\
1, & \text { otherwise }\end{cases}
\end{aligned}
$$

and

$$
\begin{aligned}
& \left(I_{F D} \bigwedge I_{\left.S_{P}, N_{E x 3}\right)(x, y)=T_{M}\left(I_{F D}(x, y), I_{S_{P}, N_{E x 3}}(x, y)\right)}\right. \\
& = \begin{cases}1, & \text { if } x \in[0,0.5) \text { and } x \leq y \\
\min \{1, \max \{1-x, y\}\}, & \text { if } x \in[0,0.5) \text { and } x>y \\
\min \{1,0.3+0.7 \cdot y\}, & \text { if } x \in[0.5,1) \text { and } x \leq y \\
\min \{\max \{1-x, y\}, 0.3+0.7 \cdot y\} & \text { if } x \in[0.5,1) \text { and } x>y \\
y, & \text { if } x=1\end{cases} \\
& = \begin{cases}\max \{1-x, y\}, & \text { if } x \in[0,0.5) \text { and } x>y \\
0.3+0.7 \cdot y, & \text { if } x \in[0.5,1) \text { and } x \leq y \\
\min \{\max \{1-x, y\}, 0.3+0.7 \cdot y\} & \text { if } x \in[0.5,1) \text { and } x>y \\
y, & \text { if } x=1 \\
1, & \text { otherwise }\end{cases}
\end{aligned}
$$

Both, $I=I_{F D} \bigvee I_{S_{P}, N_{E x 3}}$ and $J=I_{F D} \wedge I_{S_{P}, N_{E x 3}}$ violate (EP), since

$$
\begin{aligned}
& I(0.8, I(0.5,0.2))=0.65 \neq 0.608=I(0.5, I(0.8,0.2)) \text { and } \\
& J(0.4, J(0.5,0.3))=1 \neq 0.72=J(0.5, J(0.4,0.3)) .
\end{aligned}
$$

Despite of the above counterexamples, there are some special cases, where the lattice operations preserve (EP). These are the cases we use at least one fuzzy negation $N$ which has trivial range, i.e., $N(x) \in\{0,1\}$ for all $x \in[0,1]$.

Proposition 2. Let $I_{S_{1}, N_{1}}$ and $I_{S_{2}, N^{\alpha}}$ be two $(S, N)$ - implications. Then, the fuzzy implications $I_{S_{1}, N_{1}} \bigvee I_{S_{2}, N^{\alpha}}$ and $I_{S_{1}, N_{1}} \wedge I_{S_{2}, N^{\alpha}}$ satisfy (EP). 
Proof. For all $x, y, z \in[0,1]$ it is

$$
\begin{aligned}
\left(I_{S_{1}, N_{1}} \bigvee I_{S_{2}, N^{\alpha}}\right)(y, z) & =S_{M}\left(I_{S_{1}, N_{1}}(y, z), I_{S_{2}, N^{\alpha}}(y, z)\right) \\
& \stackrel{(8)}{=}\left\{\begin{array}{l}
\max \left\{S_{1}\left(N_{1}(y), z\right), z\right\}, \text { if } y \geq \alpha \\
\max \left\{I_{S_{1}, N_{1}}(y, z), 1\right\}, \text { if } y<\alpha
\end{array}\right. \\
& = \begin{cases}S_{1}\left(N_{1}(y), z\right), & \text { if } y \geq \alpha \text { (see [5] Proposition } 9) \\
1, & \text { if } y<\alpha\end{cases}
\end{aligned}
$$

Thus,

$$
\begin{aligned}
& \left(I_{S_{1}, N_{1}} \bigvee I_{S_{2}, N^{\alpha}}\right)\left(x,\left(I_{S_{1}, N_{1}} \bigvee I_{S_{2}, N^{\alpha}}\right)(y, z)\right) \\
& = \begin{cases}\left(I_{S_{1}, N_{1}} \bigvee I_{S_{2}, N^{\alpha}}\right)\left(x, S_{1}\left(N_{1}(y), z\right)\right), & \text { if } y \geq \alpha \\
\left(I_{S_{1}, N_{1}} \bigvee I_{S_{2}, N^{\alpha}}\right)(x, 1), & \text { if } y<\alpha\end{cases} \\
& = \begin{cases}S_{1}\left(N_{1}(x), S_{1}\left(N_{1}(y), z\right)\right), & \text { if } x \geq \alpha \text { and } y \geq \alpha \\
1, & \text { if } x<\alpha \text { and } y \geq \alpha \\
S_{M}\left(S_{1}\left(N_{1}(x), 1\right), S_{2}\left(N^{\alpha}(x), 1\right)\right), & \text { if } y<\alpha\end{cases} \\
& = \begin{cases}S_{1}\left(N_{1}(x), S_{1}\left(N_{1}(y), z\right)\right) & \text { if } x \geq \alpha \text { and } y \geq \alpha \\
1, & \text { if } x<\alpha \text { and } y \geq \alpha \\
1, & \text { if } y<\alpha\end{cases} \\
& = \begin{cases}S_{1}\left(N_{1}(x), S_{1}\left(N_{1}(y), z\right)\right), & \text { if } x \geq \alpha \text { and } y \geq \alpha \\
1, & \text { otherwise }\end{cases}
\end{aligned}
$$

By swapping $x$ and $y$ we have

$$
\begin{aligned}
& \left(I_{S_{1}, N_{1}} \bigvee I_{S_{2}, N^{\alpha}}\right)\left(y,\left(I_{S_{1}, N_{1}} \bigvee I_{S_{2}, N^{\alpha}}\right)(x, z)\right) \\
& = \begin{cases}S_{1}\left(N_{1}(y), S_{1}\left(N_{1}(x), z\right)\right), & \text { if } y \geq \alpha \text { and } x \geq \alpha \\
1, & \text { otherwise }\end{cases} \\
& \stackrel{(S 1)}{=} \begin{cases}S_{1}\left(N_{1}(y), S_{1}\left(z, N_{1}(x)\right)\right) & \text { if } y \geq \alpha \text { and } x \geq \alpha \\
1, & \text { otherwise }\end{cases} \\
& \stackrel{(S 2)}{=} \begin{cases}S_{1}\left(S_{1}\left(N_{1}(y), z\right), N_{1}(x)\right) & \text { if } y \geq \alpha \text { and } x \geq \alpha \\
1, & \text { otherwise }\end{cases} \\
& \stackrel{(S 1)}{=} \begin{cases}S_{1}\left(N_{1}(x), S_{1}\left(N_{1}(y), z\right)\right) & \text { if } x \geq \alpha \text { and } y \geq \alpha \\
1, & \text { otherwise }\end{cases} \\
& =\left(I_{S_{1}, N_{1}} \bigvee I_{S_{2}, N^{\alpha}}\right)\left(x,\left(I_{S_{1}, N_{1}} \bigvee I_{S_{2}, N^{\alpha}}\right)(y, z)\right) .
\end{aligned}
$$

Thus, $I_{S_{1}, N_{1}} \bigvee I_{S_{2}, N^{\alpha}}$ satisfies (EP).

Similarly for the meet, it is

$$
\begin{aligned}
\left(I_{S_{1}, N_{1}} \bigwedge I_{S_{2}, N^{\alpha}}\right)(y, z) & =T_{M}\left(I_{S_{1}, N_{1}}(y, z), I_{S_{2}, N^{\alpha}}(y, z)\right) \\
& \stackrel{(8)}{=}\left\{\begin{array}{l}
\min \left\{S_{1}\left(N_{1}(y), z\right), z\right\}, \text { if } y \geq \alpha \\
\min \left\{S_{1}\left(N_{1}(y), z\right), 1\right\}, \text { if } y<\alpha
\end{array}\right. \\
& = \begin{cases}z, & \text { if } y \geq \alpha \text { (see [5] Proposition } 9) \\
S_{1}\left(N_{1}(y), z\right), & \text { if } y<\alpha\end{cases}
\end{aligned}
$$


Thus,

$$
\begin{aligned}
& \left(I_{S_{1}, N_{1}} \bigwedge I_{S_{2}, N^{\alpha}}\right)\left(x,\left(I_{S_{1}, N_{1}} \bigwedge I_{S_{2}, N^{\alpha}}\right)(y, z)\right) \\
& = \begin{cases}\left(I_{S_{1}, N_{1}} \bigwedge I_{S_{2}, N^{\alpha}}\right)(x, z), & \text { if } y \geq \alpha \\
\left(I_{S_{1}, N_{1}} \bigwedge I_{S_{2}, N^{\alpha}}\right)\left(x, S_{1}\left(N_{1}(y), z\right)\right), & \text { if } y<\alpha\end{cases} \\
& = \begin{cases}z, & \text { if } x \geq \alpha \text { and } y \geq \alpha \\
S_{1}\left(N_{1}(x), z\right), & \text { if } x<\alpha \text { and } y \geq \alpha \\
S_{1}\left(N_{1}(y), z\right), & \text { if } x \geq \alpha \text { and } y<\alpha \\
S_{1}\left(N_{1}(x), S_{1}\left(N_{1}(y), z\right)\right), & \text { if } x<\alpha \text { and } y<\alpha\end{cases}
\end{aligned}
$$

By swapping $x$ and $y$ we have

$$
\begin{aligned}
& \left(I_{S_{1}, N_{1}} \bigwedge I_{S_{2}, N^{\alpha}}\right)\left(y,\left(I_{S_{1}, N_{1}} \bigwedge I_{S_{2}, N^{\alpha}}\right)(x, z)\right) \\
& = \begin{cases}z, & \text { if } y \geq \alpha \text { and } x \geq \alpha \\
S_{1}\left(N_{1}(y), z\right), & \text { if } y<\alpha \text { and } x \geq \alpha \\
S_{1}\left(N_{1}(x), z\right), & \text { if } y \geq \alpha \text { and } x<\alpha \\
S_{1}\left(N_{1}(y), S_{1}\left(N_{1}(x), z\right)\right), & \text { if } y<\alpha \text { and } x<\alpha\end{cases} \\
& \stackrel{(S 1)}{=} \begin{cases}z, & \text { if } x \geq \alpha \text { and } y \geq \alpha \\
S_{1}\left(N_{1}(y), z\right), & \text { if } x \geq \alpha \text { and } y<\alpha \\
S_{1}\left(N_{1}(x), z\right), & \text { if } x<\alpha \text { and } y \geq \alpha \\
S_{1}\left(N_{1}(y), S_{1}\left(z, N_{1}(x)\right)\right), & \text { if } x<\alpha \text { and } y<\alpha\end{cases} \\
& \stackrel{(S 2)}{=} \begin{cases}z, & \text { if } x \geq \alpha \text { and } y \geq \alpha \\
S_{1}\left(N_{1}(x), z\right), & \text { if } x<\alpha \text { and } y \geq \alpha \\
S_{1}\left(N_{1}(y), z\right), & \text { if } x \geq \alpha \text { and } y<\alpha \\
S_{1}\left(S_{1}\left(N_{1}(y), z\right), N_{1}(x)\right), & \text { if } x<\alpha \text { and } y<\alpha\end{cases} \\
& \stackrel{(S 1)}{=} \begin{cases}z, & \text { if } x \geq \alpha \text { and } y \geq \alpha \\
S_{1}\left(N_{1}(x), z\right), & \text { if } x<\alpha \text { and } y \geq \alpha \\
S_{1}\left(N_{1}(y), z\right), & \text { if } x \geq \alpha \text { and } y<\alpha \\
S_{1}\left(N_{1}(x), S_{1}\left(N_{1}(y), z\right)\right), & \text { if } x<\alpha \text { and } y<\alpha\end{cases} \\
& =\left(I_{S_{1}, N_{1}} \bigwedge I_{S_{2}, N^{\alpha}}\right)\left(x,\left(I_{S_{1}, N_{1}} \bigwedge I_{S_{2}, N^{\alpha}}\right)(y, z)\right) .
\end{aligned}
$$

Thus, $I_{S_{1}, N_{1}} \bigwedge I_{S_{2}, N^{\alpha}}$ satisfies (EP).

Proposition 3. Let $I_{S_{1}, N_{1}}$ and $I_{S_{2}, N_{\alpha}}$ be two $(S, N)$ - implications. Then, the fuzzy implications $I_{S_{1}, N_{1}} \bigvee I_{S_{2}, N_{\alpha}}$ and $I_{S_{1}, N_{1}} \wedge I_{S_{2}, N_{\alpha}}$ satisfy (EP).

Proof. The proof is omitted because it is similar to the proof of Proposition 2.

Although, the proofs of the Propositions 2 and 3 could also been deduced by Proposition 4.1 in [7] (see also [8] Proposition 5.2, [9] Proposition 5.1), an alternative proof has been presented. That is because the induced formula of lattice operations (join and meet) should also been mentioned. Moreover, Propositions 2 and 3 hold for an $(\mathrm{S}, \mathrm{N})$ - implication, whose negation $N$ has trivial range and any other fuzzy implication $I[7-9]$, but this is out of the purpose of this paper, since we study only $(\mathrm{S}, \mathrm{N})$ - implications. 


\section{Conclusions}

In this paper, we have investigated the solution of a specific case of an open problem (see [1] Remark 6.1.5, [6] Problem 3.1) that is related to the preservation of the exchange principle (EP) of fuzzy implications via lattice operations. We have investigated these solutions with the use of only $(\mathrm{S}, \mathrm{N})$ - implications. We have presented sufficient, but not necessary conditions for this preservation. More specific, the conclusions are the following: It is ensured that (EP) is preserved via the lattice operations, when two $(\mathrm{S}, \mathrm{N})$ - implications generated from the same t-conorm $S$ or at least one of them generated from a fuzzy negation $N$, which has trivial range. Moreover, the same result holds, when two $(\mathrm{S}, \mathrm{N})-$ implications, $I_{S_{1}, N_{1}}$ and $I_{S_{2}, N_{2}}$, generated from comparable t-conorms and fuzzy negations, such that $S_{1} \leq S_{2}$ and $N_{1} \leq N_{2}$. On the other hand, we have presented counterexamples (Examples 1, 2, 3 and 4) that proved the violation of (EP) in general, in many other cases. However, this problem needs more investigation. Our intention is to study this problem in detail in the near future.

\section{References}

1. Baczyński, M., Jayaram, B.: STUDFUZZ. Studies in Fuzziness and Soft Computing, vol. 231. Springer, Heidelberg (2008). https://doi.org/10.1007/978-3-540-69082-5

2. Dimuro, G.P., Bedregal, B., Bustince, H., Jurio, A., Baczyński, M., Mis, K.: QLoperations and QL-implication functions constructed from triples $(\mathrm{O}, \mathrm{G}, \mathrm{N})$ and the generation of fuzzy subsethood and entropy measures. Int. J. Approx. Reason. 82, 170-192 (2017)

3. Drewniak, J.: Invariant fuzzy implications. Soft. Comput. 10, 506-513 (2006). https://doi.org/10.1007/s00500-005-0526-4

4. Fodor, J.C., Roubens, M.: Fuzzy Preference Modeling and Multicriteria Decision Support. Kluwer, Dordrecht (1994)

5. Grammatikopoulos, D.S., Papadopoulos, B.K.: A method of generating fuzzy implications with specific properties. Symmetry 12(1), 155-170 (2020)

6. Mesiar, R., Stupňanová, A.: Open problems from the 12th international conference on fuzzy set theory and its applications. Fuzzy Sets Syst. 261, 112-123 (2015)

7. Vemuri, N.R.: Mutually exchangeable fuzzy implications. Inf. Sci. 317, 1-24 (2015)

8. Vemuri, N.R., Jayaram, B.: Preservation of the exchange principle under lattice operations on fuzzy implications. In: 8th International Summer School on Aggregation Operators AGOP 2015, pp. 227-232. University of Silesia, Katowice (2015)

9. Vemuri, N.R., Jayaram, B.: Lattice operations on fuzzy implications and the preservation of the exchange principle. Fuzzy Sets Syst. 301, 64-78 (2016) 